\title{
openheart Cost-utility of transcatheter aortic valve implantation for inoperable patients with severe aortic stenosis treated by medical management: a UK cost-utility analysis based on patient-level data from the ADVANCE study
}

Stephen Brecker, ${ }^{1}$ Stuart Mealing, ${ }^{2}$ Amie Padhiar, ${ }^{2}$ James Eaton, ${ }^{2}$ Mark Sculpher, ${ }^{2}$ Rachele Busca, ${ }^{3}$ Johan Bosmans, ${ }^{4}$ Ulrich J Gerckens, ${ }^{5}$ Peter Wenaweser, ${ }^{6}$ Corrado Tamburino, ${ }^{7}$ Sabine Bleiziffer, ${ }^{8}$ Nicolo Piazza, ${ }^{9}$ Neil Moat, ${ }^{10}$ Axel Linke ${ }^{11}$

To cite: Brecker S,

Mealing S, Padhiar A, et al. Cost-utility of transcatheter aortic valve implantation for inoperable patients with severe aortic stenosis treated by medical management: a UK cost-utility analysis based on patient-level data from the ADVANCE study. Open Heart 2014;: : 000155 .

doi:10.1136/openhrt-2014000155

- Additional material is available. To view please visit the journal (http://dx.doi.org/ 10.1136/openhrt-2014000155)

Received 9 June 2014 Revised 18 August 2014 Accepted 1 September 2014

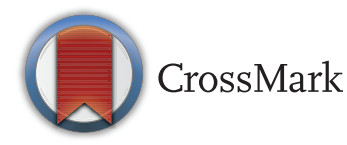

For numbered affiliations see end of article.

Correspondence to Dr Stuart Mealing; stuart.mealing@iconplc.com

\section{ABSTRACT}

Objective: To use patient-level data from the ADVANCE study to evaluate the cost-effectiveness of transcatheter aortic valve implantation (TAVI) compared to medical management (MM) in patients with severe aortic stenosis from the perspective of the UK NHS.

Methods: A published decision-analytic model was adapted to include information on TAVI from the ADVANCE study. Patient-level data informed the choice as well as the form of mathematical functions that were used to model all-cause mortality, health-related quality of life and hospitalisations. TAVI-related resource use protocols were based on the ADVANCE study. MM was modelled on publicly available information from the PARTNER-B study. The outcome measures were incremental cost-effectiveness ratios (ICERs) estimated at a range of time horizons with benefits expressed as quality-adjusted life-years (QALY). Extensive sensitivity/ subgroup analyses were undertaken to explore the impact of uncertainty in key clinical areas.

Results: Using a 5-year time horizon, the ICER for the comparison of all ADVANCE to all PARTNER-B patients was $£ 13943$ per QALY gained. For the subset of ADVANCE patients classified as high risk (Logistic EuroSCORE $>20 \%$ ) the ICER was $£ 17718$ per QALY gained). The ICER was below $£ 30000$ per QALY gained in all sensitivity analyses relating to choice of MM data source and alternative modelling approaches for key parameters. When the time horizon was extended to 10 years, all ICERs generated in all analyses were below $£ 20000$ per QALY gained. Conclusion: TAVI is highly likely to be a cost-effective treatment for patients with severe aortic stenosis.

\section{INTRODUCTION}

Transcatheter aortic valve implantation (TAVI) has become the standard of care for patients with severe symptomatic aortic

\section{KEY MESSAGES}

What is already known about this subject?

- Severe symptomatic aortic stenosis in patients who cannot receive surgical aortic valve replacement carries a poor prognosis. The introduction of transcatheter aortic valve implantation has offered an opportunity for improved outcomes in this patient group.

What does this study add?

- This study is a cost-effectiveness analysis of TAVI using evidence from the 'real world' ADVANCE study and the CoreValve system. This is the first formal cost-effectiveness analysis using data for the CoreValve system and concludes that TAVI is likely to represent a costeffective intervention as compared to medical management.

How might this impact on clinical practice?

- In properly selected patients, TAVI offers substantial improvements in symptoms and life expectancy and is likely to represent a costeffective use of healthcare budgets.

stenosis (AS) who are considered at extreme or prohibitive risk for surgical aortic valve replacement and as an acceptable alternative to surgery for those at high risk. ${ }^{1}$ However, these treatments are expensive, with high index costs due to the expense of the prosthesis. Typical TAVI candidates are costly to care for without intervention due to repeated hospitalisation and heart failure (HF) therapies. $^{2}$ Furthermore, their quality and quantity of life is poor without treatment. ${ }^{34}$

The UK National Institute of Health and Care Excellence $(\mathrm{NICE})^{5}$ is charged with 
considering the clinical and cost-effectiveness of treatments and then with making recommendations as to their provision within the National Health Service (NHS). Cost-utility analysis assesses two or more alternative courses of action in terms of their costs and benefits. The comparison is summarised using the expected incremental cost-effectiveness ratio (ICER). This is a measure of the additional cost per additional unit of health gain produced by one intervention compared to another. NICE's preferred form of costeffectiveness analysis uses the quality-adjusted life-year (QALY) to describe the outcome of each intervention. By extension, NICE's preferred form of ICER is the cost per QALY gained.

We aimed to measure the cost-effectiveness of TAVI implantation by comparing costs and benefits of patients receiving TAVI as part of the ADVANCE study, with those receiving medical management (MM) in Cohort $\mathrm{B}$ of the PARTNER (Placement of Aortic Transcatheter Valves) study (henceforth referred to as PARTNER-B).

\section{METHODS}

\section{Data sources}

Individual patient data (IPD) from the international Medtronic CoreValve ADVANCE study were used to model costs and benefits of the TAVI cohort. ${ }^{6}$ One of the largest and most rigorous TAVI postmarket studies to date, ADVANCE comprises over 1000 patients from 44 centres in 12 countries in Western Europe, Asia and South America. From March 2010 to July 2011, 1015 patients were enrolled in the ADVANCE study, of which 996 patients underwent attempted implant with the CoreValve device. The mean age was $81.1 \pm 6.4$ years (range 51-96 years) and 51\% were female. The baseline peak and mean aortic valve gradients were $75.9 \pm 25.1$ and $45.6 \pm 15.5 \mathrm{~mm} \mathrm{Hg}$, respectively, and the mean aortic valve area was $0.7 \pm 0.3 \mathrm{~cm}^{2}$. The median $(\mathrm{Q} 1, \mathrm{Q} 3)$ logistic EuroSCORE was $16 \%(10.3,25.3 \%)$ and the median (Q1，Q3) STS score was 5.3\% (3.6, 7.8\%). Twelve months of follow-up data were used as the basis of all analyses. Patient source data were $100 \%$ monitored and events were independently adjudicated. Over 120 patients from the ADVANCE study were recruited from five UK centres; the baseline characteristics of these patients were not significantly different from the cohort overall. Data from the PARTNER-B randomised trial were used to parameterise the MM arm of the model. $^{3} 78$

\section{Model description}

We used the analytical framework of a previously published model to inform this analysis. ${ }^{9}$ Briefly, two separate Markov models were used to characterise what happens to a TAVI patient in the first 30 days postprocedure and for the remainder of their lifetime, respectively. Cycle lengths of 1 day and 1 month were applied in the short-term and long-term models, respectively. Contingent on being alive at the end of the short-term model, all individuals enter the long-term model where they remain until dead. An individual can be in the following health states: dead, alive at home, or alive in hospital for an additional TAVI-related procedure. Heart failure-related rehospitalisations are included as a cost rather than a health state and can be experienced by all patients who are alive regardless of health state. TAVI patients enter the model in the surgical procedure health state and MM patients in the alive at home health state. Parametric survival functions were fitted to the relevant mortality data and extrapolated for the time horizon specified in the model.

The model schematic is presented in figure 1. Each health state is associated with costs and utilities that patients accrue throughout the model. The accrual process is continued for a fixed time period (the cycle length) and results are expressed as lifetime costs (£UK) and benefits (QALYs). Patients were entered into the short-term model with a median starting age of 82 years, based on the mean age from the ADVANCE study. The model was constructed in Microsoft Excel and was run
Figure 1 Model schematic (TAVI, transcatheter aortic valve implantation).

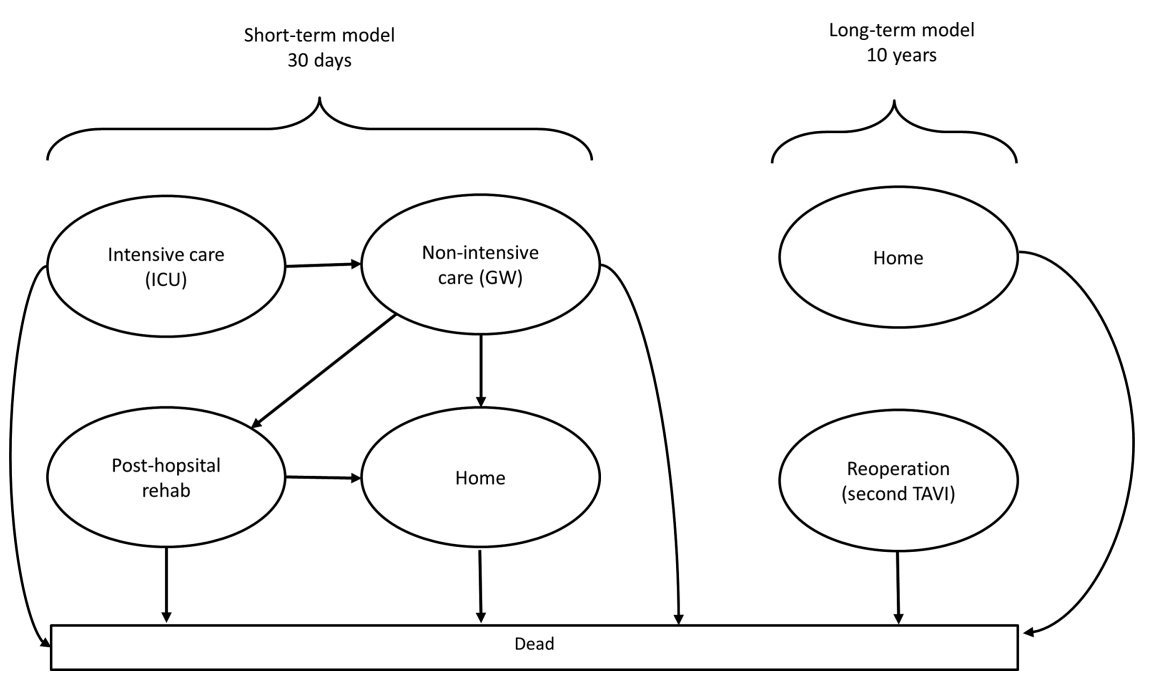


for a time horizon of 5 years. A UK NHS perspective was used with discount rates of $3.5 \%$ per annum for costs as well as utilities. ${ }^{5}$

To explore the implications of patient-level heterogeneity on cost-effectiveness, in addition to using all patients we stratified patients in the ADVANCE study into highrisk and low-risk groups defined using the reported Logistic EuroSCORE, used to calculate the predicted operative mortality for patients undergoing cardiac surgery (High/Low: split score $=20 \%)$.

\section{Estimating mortality}

A range of parametric survival functions were fitted to the time-to-event data which included: exponential, Weibull, Gompertz, log-normal, log-logistic and Gamma functions. ${ }^{10}$ The choice of distribution for the cost-utility model was based on within trial fit and clinically plausible extrapolation beyond the trial data. The former was assessed by comparing Akaike Information Criteria (AIC) and Bayesian Information Criteria (BIC), and also by examining Cox-Snell residual plots. The latter was assessed by visual assessment of the extrapolated survival beyond the trial period. The proportional hazards assumption was assessed through a log-cumulative hazard plot of the survival data and examining Schoenfeld residuals by risk group. ${ }^{10}$

\section{Estimating health-related quality of life}

Health-related quality of life (HRQoL) was included into the model as utilities using ADVANCE EQ-5D (EuroQol) values for TAVI, with reported patient-level EQ-5D scores mapped to utility weights using standard UK tariffs. ${ }^{11}$

A random intercepts multilevel model was constructed to estimate HRQoL over time using the utility weights. The model accounts for baseline utility deviating from the mean. A random effects intercept and gradient model was also investigated, which allows for differences in the trajectory of utility over time between patients in addition to differences at baseline. The chosen model was restricted to never exceed the age-adjusted and gender-adjusted EQ-5D population norms.

For patients receiving MM, a fixed utility decrement based on the PARTNER-B data was applied to the age-adjusted and gender-adjusted population norms as described in Watt et al. ${ }^{9}$

\section{Estimating hospitalisations}

In the base case, all-cause rehospitalisation rates were captured using a time-dependent function, allowing the rates to vary over time. The time-dependent rates were based on PARTNER-B data, ${ }^{8}$ where Weibull distributions for TAVI and MM were fitted independently to model the time to first rehospitalisation. This approach assumes that the differences in device choice and patient characteristics do not have an impact on hospitalisation rates. Considering the potential heterogeneity we have identified between the cohorts, we performed a sensitivity analysis on this assumption by using a fixed rate of hospitalisation derived from either ADVANCE or the literature ${ }^{12}$ in every model cycle.

\section{Resource use and unit costs}

Treatment costs were sourced from the latest British National Formulary. ${ }^{13}$ Additional costs were sourced, where available, from publicly accessible databases ${ }^{14} 15$ or from a recent UK-based analysis of TAVI carried out on behalf of NICE. ${ }^{16}$ Resource use data including time spent in different hospital departments and overall length of stay were taken from ADVANCE. In order to fully align the underlying MM hospitalisation rate and costs we have included the PARTNER-B balloon aortic valvuloplasty (BAV) rate into the model. ${ }^{3}$ Hourly procedure costs were taken from Raikou et $a l^{17}$ and inflated to current values using an appropriate inflation index. ${ }^{15}$ Key parameter inputs are presented in online supplementary tables S1/S2.

\section{Sensitivity analyses}

Detailed analyses relating to three elements of uncertainty were undertaken. First, for each of the two main comparisons a full exploration of parameter uncertainty was undertaken via probabilistic sensitivity analyses. Second, a deterministic assessment of parameter uncertainty was undertaken for the key clinical comparison (ADVANCE high risk vs all PARTNER-B MM) patients. Finally, due to the number of different approaches that could be used to model key aspects of the patient pathway (structural uncertainty), a series of scenario analyses were performed to explore the implications of alternative choices.

In addition to the analyses described above, we also assessed the sensitivity around the survival data for MM patients by exploring the use of different mortality sources from PARTNER-B: 1-year data, ${ }^{3}$ 2-year data, 3-year data, ${ }^{7}$ with and without $\mathrm{BAV}^{8}$ and the Society of Thoracic Surgery (STS) Score $<5 \%$ subgroup. $^{8}$ In addition, we used external data sources as an alternative to PARTNER-B to model mortality for MM patients. ${ }^{18} 19$ We also explored alternative assumptions relating to location of death and differences in degenerative valve disease in all HF hospitalisation events. Finally, we explored the implications of alternative choices of time horizon on cost-effectiveness.

\section{CLINICAL RESULTS \\ Patient characteristics}

Baseline characteristics in all key patient groups are presented in table 1. Highly significant differences $(p<0.001)$ were identified in all patients recruited into ADVANCE and the PARTNER-B MM arm for a number of key cardiovascular endpoints including atrial fibrillation, coronary artery disease, cerebrovascular disease, Logistic EuroSCORE and the proportion of patients with New York Heart Association (NYHA) class III or IV HF. Restricting the comparison to those in the 
Table 1 Baseline characteristics for ADVANCE and PARTNER-B medical management

\begin{tabular}{|c|c|c|c|c|c|}
\hline & \multicolumn{2}{|l|}{ ADVANCE } & \multirow{2}{*}{$\begin{array}{l}\text { PARTNER-B } \\
\text { Medical } \\
\text { management }\end{array}$} & \multicolumn{2}{|c|}{$\begin{array}{l}\text { p Values (ADVANCE vs } \\
\text { PARTNER-B) }\end{array}$} \\
\hline & $\begin{array}{l}\text { All } \\
\text { patients }\end{array}$ & $\begin{array}{l}\text { High-risk cohort } \\
\text { (EuroSCORE>20\%) }\end{array}$ & & $\begin{array}{l}\text { All } \\
\text { patients }\end{array}$ & $\begin{array}{l}\text { High-risk } \\
\text { cohort }\end{array}$ \\
\hline$N$ & 1015 & 369 & 179 & & \\
\hline Mean age in years $( \pm S D)$ & $81.1 \pm 6.4$ & $82.7 \pm 5.7$ & $83.0 \pm 8.0$ & 0.001 & 0.614 \\
\hline Male (\%) & 49.4 & 49.9 & 46.9 & 0.537 & 0.510 \\
\hline Logistic EuroSCORE, \% ( \pm SD) & $19.4 \pm 12.3$ & $32.3 \pm 11.0$ & $30.4 \pm 19.1$ & $<0.001$ & 0.141 \\
\hline NYHA III/IV (\%) & 79.6 & 84.6 & 93.9 & $<0.001$ & 0.002 \\
\hline Diabetes (\%) & 31.3 & 27.1 & Not reported & NA & NA \\
\hline Coronary artery disease (\%) & 57.6 & 66.1 & 74.3 & $<0.001$ & 0.052 \\
\hline Peripheral vascular disease (\%) & 19.5 & 27.1 & 25.1 & 0.086 & 0.617 \\
\hline Atrial fibrillation (\%) & 32.9 & 36.8 & 48.8 & $<0.001$ & 0.005 \\
\hline Cerebrovascular disease (\%) & 12.9 & 17.6 & 27.5 & $<0.001$ & 0.007 \\
\hline Prior MI (\%) & 16.0 & 22.0 & 26.4 & 0.001 & 0.289 \\
\hline Prior PCI (\%) & 31.1 & 36.0 & 24.8 & 0.090 & 0.009 \\
\hline Prior CABG (\%) & 21.4 & 34.7 & 45.6 & $<0.001$ & 0.014 \\
\hline Prior permanent pacemaker (\%) & 12.9 & 16.5 & 19.5 & 0.017 & 0.384 \\
\hline
\end{tabular}

CABG, coronary artery bypass graft; MI, myocardial infarction; NYHA, New York Heart Association; PCI, percutaneous coronary intervention.

ADVANCE high-risk group, fewer significant differences were observed and there were no longer any significant differences in logistic EuroSCORE, prior myocardial infarction (MI) and prior pacemaker usage. While not an element of this analysis, the differences between the TAVI arm of the PARTNER-B study and the ADVANCE high-risk group were even less pronounced. Differences in Logistic EuroSCORE (\%), coronary artery disease $(\%)$ and atrial fibrillation (\%) were 26.4 vs $32.3,67.6$ vs 66.1 and 32.9 vs 36.3 for the TAVI arm of the PARTNER-B study and the ADVANCE high-risk group, respectively. ${ }^{6} 7$

\section{Predicted survival}

There was strong evidence to suggest the proportional hazards assumption was violated for treatment-specific all risk groups, and hence independent survival curves

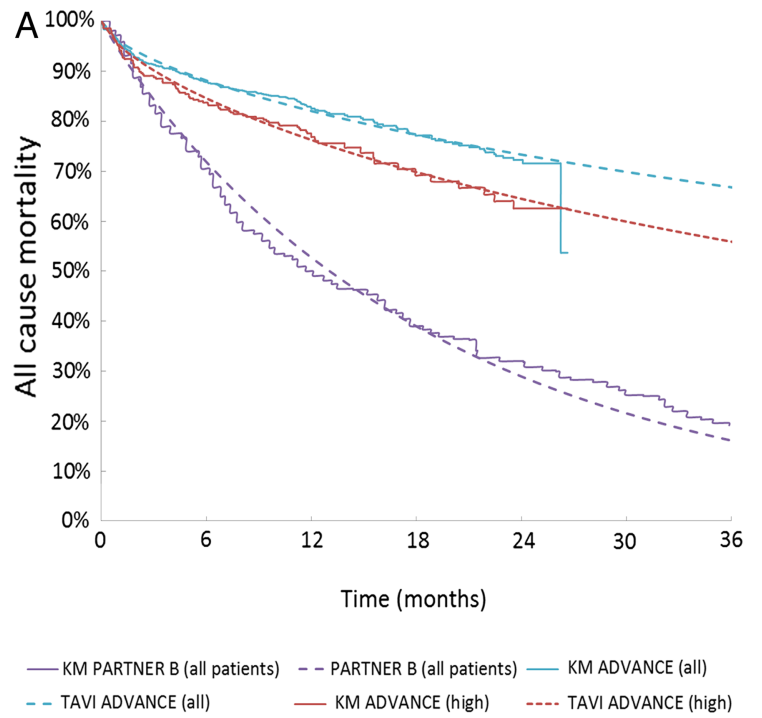

were used. The Weibull function was chosen to model survival for all patient groups (see online supplementary table S3).

Alongside the function for all patients, these survival functions were restricted to not exceed the age-specific and gender-specific population survival sourced from UK life tables. ${ }^{20}$ A comparison of the raw and fitted data is presented in figure 2A. Based on the ADVANCE highrisk cohort, 1-year survival for TAVI patients was $77 \%$ and 5-year survival $44 \%$. For all ADVANCE patients the predicted 1-year and 5-year survival estimates were $83 \%$ and $56 \%$, respectively. In comparison, MM patients had a 53\% chance of survival at 1 year and a $5 \%$ chance of surviving to 5 years, using the 3 years PARTNER-B data (figure 2B).

When expressed using a numbers needed to treat (NNT) metric the value generated using the 5-year time

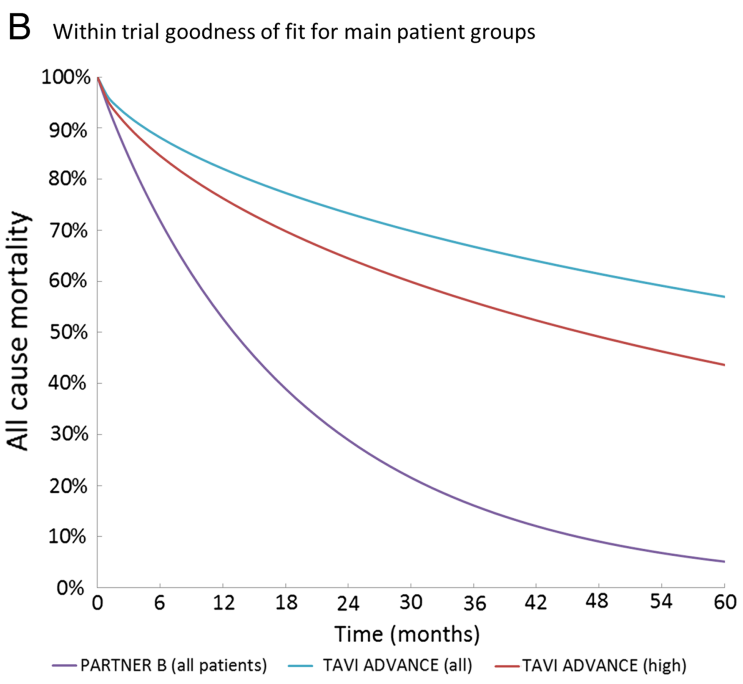

long term extrapolation for all main patient groups

Figure 2 (A) Within trial goodness of fit for main patient groups. (B) Long-term extrapolation for all main patient groups (TAVI, transcatheter aortic valve implantation). 


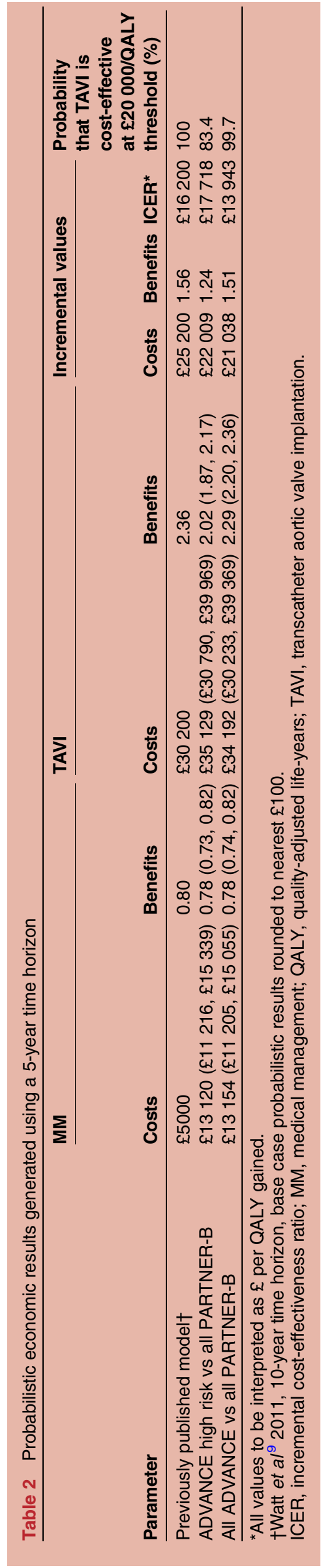

horizon for all ADVANCE patients compared to all PARTNER-B MM is 2.0. The corresponding value for the comparison of the ADVANCE high-risk group to all PARTNER-B MM patients is 2.6.

\section{Other clinical endpoints}

A random effects intercept model was chosen to estimate utility over time for TAVI patients based on the ADVANCE data (see online supplementary table S5). The model predicted a monthly utility increase of 0.002 (95\% CI -0.002 to 0.006$)$.

Using the time-dependent hospitalisation function, estimated from the PARTNER-B data, the predicted probability of HF-hospitalisation in the first year is lower for TAVI patients (18.4\%) compared to MM patients $(44.5 \%)$, using the ADVANCE high-risk group for all patients receiving TAVI and all PARTNER-B patients for the MM mortality data. Using the ADVANCE data a fixed rate of 0.668 events per month was estimated and was applied as a sensitivity analysis in the TAVI arm.

\section{Cost-utility results}

The probabilistic cost-effectiveness results are presented in table 2 and the cost-effectiveness plane for both comparisons is presented in figure 3. The ICER for the most optimistic comparison (all ADVANCE vs all PARTNER-B) and also the most clinically plausible comparison (ADVANCE high risk vs all PARTNER-B) are below the threshold values used by NICE in their decision-making process ( $£ 20000$ per QALY gained) and in both cases, TAVI was more likely than not to be cost-effective (minimum probability $=83.4 \%$ ). The corresponding values generated using a longer time horizon (10 years) are presented in the online supplementary table S6 with all ICERs being lower than $£ 13000$ per QALY gained.

The results generated using the ADVANCE high-risk data for TAVI, but parameterised solely using a range of data from the PARTNER-B study as well as two other historical AS publications are reported in table 3 (time horizon $=5$ years) and online supplementary table S7 (time horizon $=10$ years). In only 3 of 16 analyses was the ICER above $£ 20000$ per QALY gained (max value: $£ 27$ 790). With the exception of the clinically implausible comparison of ADVANCE high-risk and PARTNER-B low-risk patients, all ICERs generated using PARTNER-B survival data are below $£ 20000$ per QALY gained.

\section{Sensitivity analyses}

The parameters in the model that had the greatest impact on the ICER are presented in figure 4. The model was most sensitive to changes in the cost of an MM HF hospitalisation event. Changing the acquisition cost of TAVI by $\pm 15 \%$, or assuming that everyone in the TAVI arm required additional pacing, did not generate an ICER in excess of $£ 20000$ per QALY gained. 
Figure 3 Cost-effectiveness plane for all PARTNER-B vs all ADVANCE and all PARTNER vs ADVANCE high risk comparisons with ICERs from five studies; dashed line represents willingness to pay of $£ 20000$ per QALY gained (ICER, incremental cost-effectiveness ratio; MM, medical management; QALY, quality-adjusted life-years; CABG, coronary artery bypass graft; HF, heart failure; CRT, cardiac resynchronization therapy).

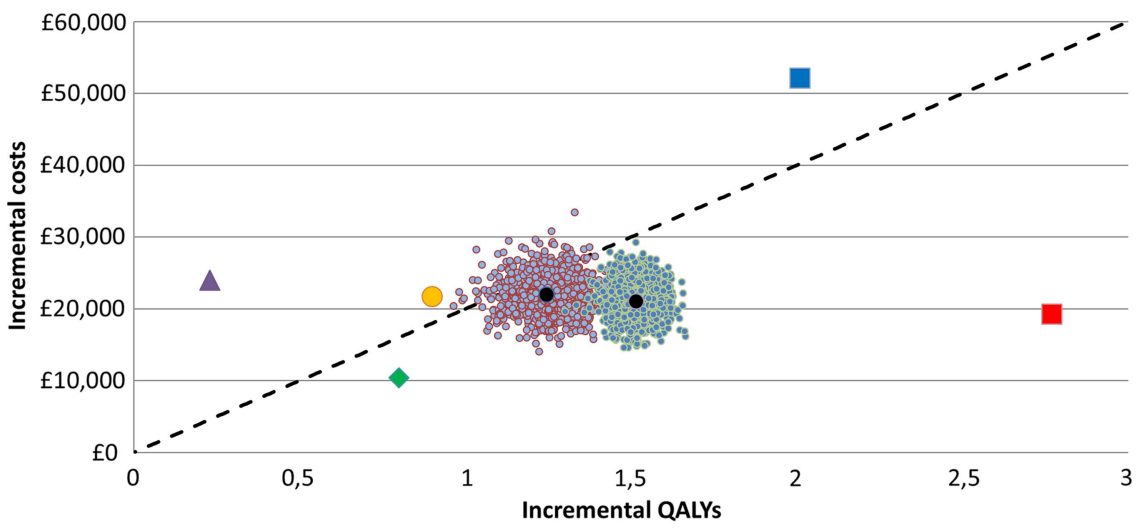

- All ADVANCE vs All PARTNER

\ Sunitinib Renal Cancer

- CRT-D vs. OPT (80yr old)

- CABG vs. MM (80yr old)

- High risk ADVANCE vs All PARTNER mean ICER
- All ADVANCE vs All PARTNER mean ICER

- CRT in mild HF

- Imatinib CML

- High risk ADVANCE vs All PARTNER
The ICERs generated using fixed hospitalisation rates or fixed utility decrements are below the NICE costeffectiveness threshold with the maximum value generated being £18577 per QALY gained (table 4, see online supplementary table S8). Compared to the base case results in table 2 , the impact of using the alternative modelling approaches was negligible.

The base case analysis assumed that $50 \%$ of patients died in a hospital environment. When the more conservative assumption was made that nobody died in a hospital based environment, the impact on the ICER for the two main clinical comparisons was an increase in the ICER of approximately $10 \%$ (table 4 , see online supplementary table S8). This increase did not result in any of the ICERs being above the NICE cost-effectiveness threshold (maximum value $£ 19481$ per QALY gained).

Based on the clinical protocols presented in Orlando et $a l,{ }^{16}$ we assumed a difference in the cost of treating an HF hospitalisation event in both arms. When the TAVI value is used in the MM arm, the impact on the ICER is an increase of approximately $7 \%$ (table 4 , see online supplementary table S8). The ICERs for both comparisons again remained below the NICE cost- effectiveness threshold (maximum value £18 883 per QALY gained).

\section{DISCUSSION}

Significant advances in medical devices are often approached with great anticipation and substantial reservations. Following the introduction of TAVI in 2007, its uptake by the clinical community has been remarkable, ${ }^{21}$ yet until the publication of the PARTNER-B study in 2010 there was no comparative evidence of the clinical and cost-effectiveness of TAVI. Understandably, the reaction by clinicians and payers has been mixed; while some have openly welcomed $\mathrm{TAVI}^{22}$ others have voiced caution and concern at its widespread adoption. ${ }^{23} 24$

With the publication of the PARTNER-B study and its encore publications, the clinical and economic case for TAVI in patients for whom surgical aortic valve replacement (SAVR) is not an option now seems clearer. ${ }^{3} 82526$ However, the PARTNER-B study was initiated in 2007, and since then there have been many advances in device, placement and patient selection, such that the patient population selected for PARTNER (especially

Table 3 Sensitivity analysis: exploring alternative MM mortality data sources

\begin{tabular}{|c|c|c|c|c|c|c|c|}
\hline \multirow[b]{2}{*}{ Parameter } & \multicolumn{2}{|l|}{ MM } & \multicolumn{2}{|l|}{ TAVI } & \multicolumn{3}{|c|}{ Incremental values } \\
\hline & Costs & Benefits & Costs & Benefits & Costs & Benefits & ICER $^{*}$ \\
\hline PARTNER-B (1 year) & $£ 12972$ (£11 080, £15 092) & $0.75(0.70,0.80)$ & $£ 35160(£ 30736, £ 40248)$ & $2.02(1.87,2.17)$ & $£ 22188$ & 1.27 & $£ 17483$ \\
\hline PARTNER-B (2 years) & $£ 12679(£ 10763, £ 14669)$ & $0.71(0.67,0.75)$ & $£ 35156$ (£30 830, £39 808) & $2.02(1.88,2.16)$ & $£ 22478$ & 1.31 & $£ 17165$ \\
\hline PARTNER-B (3 years) & $£ 13120(£ 11216, £ 15339)$ & $0.78(0.73,0.82)$ & $£ 35129$ (£30 790, £39 969) & $2.02(1.87,2.17)$ & $£ 22009$ & 1.24 & $£ 17718$ \\
\hline PARTNER-B no BAV† & $£ 9233(£ 7536, £ 10938)$ & $0.69(0.66,0.73)$ & $£ 35173(£ 30519, £ 40209)$ & $2.02(1.87,2.17)$ & $£ 25940$ & 1.33 & $£ 19500$ \\
\hline PARTNER-B with BAV $\dagger$ & $£ 13268$ (£11 311, £15 429) & $0.69(0.65,0.73)$ & $£ 35214$ (£30 820, £40 434) & $2.02(1.87,2.16)$ & $£ 21946$ & 1.33 & $£ 16441$ \\
\hline PARTNER-B STS $<5 \%$ & $£ 16148(£ 13846, £ 18881)$ & $1.33(1.27,1.40)$ & $£ 35222$ (£30 740, £40 378) & $2.02(1.86,2.17)$ & $£ 19074$ & 0.69 & $£ 27790$ \\
\hline Varadarajan et al & $£ 14734(£ 12607, £ 17054)$ & $1.09(1.02,1.16)$ & $£ 35077$ (£30 077, £40 329) & $2.02(1.86,2.17)$ & $£ 20344$ & 0.93 & $£ 21849$ \\
\hline Bouma et al & $£ 16338$ (£13 929, £19 119) & $1.38(1.30,1.45)$ & $£ 35139$ (£30 734, £40 192) & $2.02(1.87,2.17)$ & $£ 18751$ & 0.86 & $£ 20667$ \\
\hline
\end{tabular}

Data presented as mean, $95 \%$ Crl. Time horizon $=5$ years. TAVI arm based on ADVANCE high-risk group.

${ }^{*}$ All values to be interpreted as $£$ per QALY gained.

†Costings altered to reflect alternative assumptions about BAV usage.

$\mathrm{BAV}$, balloon aortic valvuloplasty; Crl, credible interval; ICER, incremental cost-effectiveness ratio; MM, medical management; QALY, quality-adjusted life-years; TAVI, transcatheter aortic valve implantation. 


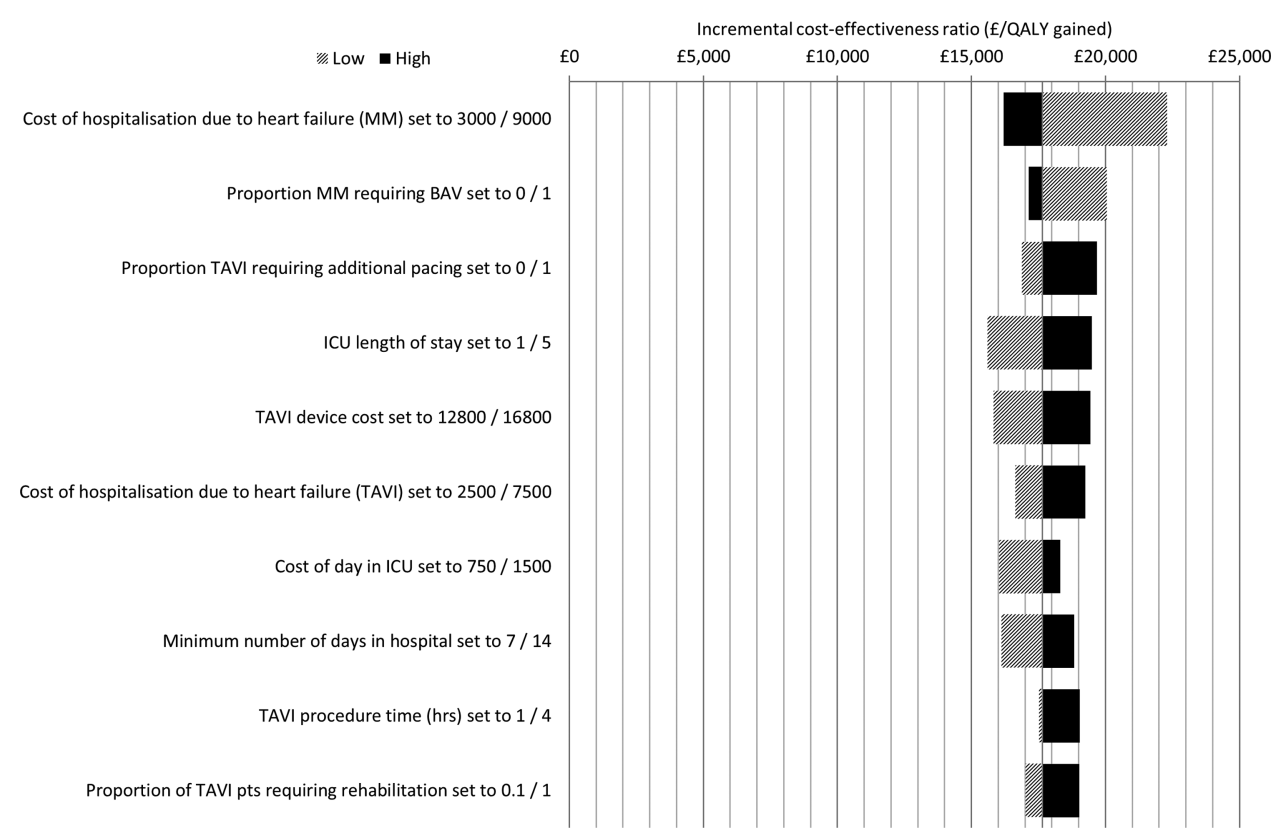

Figure 4 Deterministic univariate sensitivity analyses (BAV, balloon aortic valvuloplasty; ICU, intensive care unit; MM, medical management; QALY, quality-adjusted life-years; TAVI, transcatheter aortic valve implantation).

Cohort B) no longer represents those treated in clinical practice. ADVANCE is the first study of the Medtronic CoreValve system and TAVI to have been published since PARTNER-B. A monitored, independently adjudicated 'real world' single arm study, ADVANCE was designed to evaluate the safety and efficacy of TAVI in 1015 patients considered inoperable or at high risk for SAVR.

The ADVANCE study provides good-quality data to explore the efficacy and cost-effectiveness of the CoreValve system. However, as a single arm study there is no common comparator with which we could conduct quantitative evidence synthesis across trials, so we have used naive study comparisons to compare treatment with the CoreValve system alongside MM. Such comparisons are limited in their ability to compare across trials, as there can be many confounding factors, such as differences in the baseline characteristics and trial protocols, which may introduce bias to estimates of comparative effectiveness. One way to accommodate for this bias is to compare like with like using patient-level data from all relevant trials.

Without access to the IPD from comparator studies we have characterised uncertainty in the prognosis of patients who did not get TAVI by using publicly available subgroup data from the PARTNER-B cohort to inform a range of sensitivity analyses. In all of these we have, by necessity, assumed that the resource use protocols from the main PARTNER-B cohort, in particular the hospitalisation rates, are directly applicable to all subgroups. This is a key limitation of the analyses.

Despite this limitation, using the available data and a truncated time horizon ( 5 years), our analysis shows that TAVI is highly likely to be a cost-effective use of UK healthcare resources in inoperable patients with AS. Compared to all patients recruited into PARTNER-B, individuals in the high-risk ADVANCE cohort (the most comparable population to the PARTNER-B cohort) received an increase of 1.24 QALYs at an additional cost to the healthcare system of $£ 22009$. The ICER was thus $£ 17718$ per QALY gained and the probability of being cost-effective at a threshold value of $£ 20000$ per QALY gained of $83.4 \%$.

Increasing the time horizon that the model is run for to an approximate patient lifetime (10 years) results in the ICER falling to $£ 12003$ per QALY gained (100\% cost-effective at the UK decision threshold). Importantly, the ICERs generated by this analysis are broadly consistent with other published models of TAVI, ${ }^{95-30}$ and more conservative than the estimates produced by an independent UK-based Health Technology Assessment (HTA) of TAVI. ${ }^{16}$

In reporting the results of this analysis we have considered and presented ICERs over two distinct time horizons, 5 and 10 years. In the conduct of cost-utility analysis it is necessary and often required by HTA bodies that ICERs are presented over the patient's lifetime; for our purposes and given the starting age of the population, 10 years was justified as being sufficient to meet this requirement. However, researchers are often sceptical of analyses that extrapolate overall survival from limited trial follow-up, so we have also presented our analyses using a 5-year time horizon. Our current knowledge from the PARTNER-B cohort informs us that over the 3-year follow-up of the trial, overall survival in both patient cohorts was predictable and readily lends itself to extrapolation. As would be expected, the time horizon does impact on the ICER, 


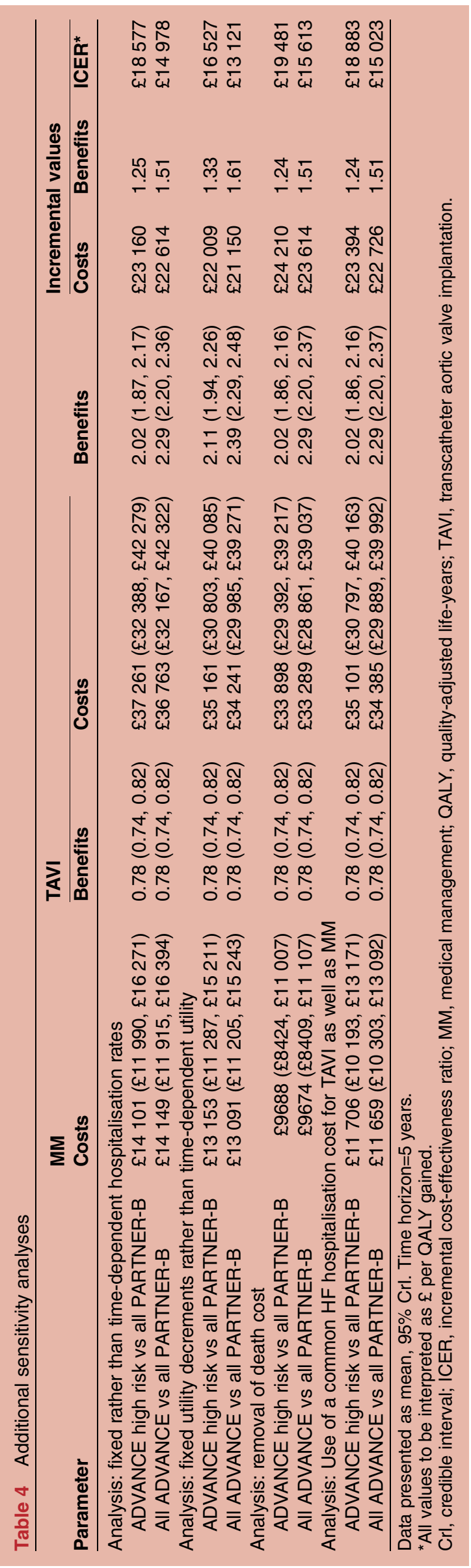

though in all cases the additional 5 years increases the QALY gain by less than a third resulting in more favourable ICERs under all scenario analyses, which are consistent with the original Watt publication. ${ }^{9}$

In developing this model we have attempted to provoke an informed discussion on the likely costeffectiveness of TAVI in inoperable patients. To achieve this we have used the ADVANCE IPD for the CoreValve system alongside the PARTNER trial and other literature to generate a large number of scenario analyses, almost all of which show TAVI to be cost-effective in inoperable patients. This study gives reassurance that TAVI and the CoreValve system is likely to be a cost-effective intervention for inoperable patients.

Putting the results of this UK cost-utility analysis into context, we have included the ICERs of a small number of other interventions approved by NICE in recent history in figure 3 . It has been stated in the literature that TAVI in inoperable patients may only be a palliative treatment. ${ }^{30}$ However, TAVI is associated with substantial improvements in overall survival and QALYs when compared to agents such as sunitinib for renal cancer and has a better QALY gain than cardiac resynchronisation therapy vs optimal pharmacological therapy in elderly patients ( $>80$ years). When considered against the spectrum of decisions made by agencies such as NICE, TAVI represents value for money.

The role of economic evaluations in decision-making is to inform, not dictate decisions about which healthcare interventions to fund. In such analyses, researchers attempt to incorporate all the appropriate evidence into an analysis to compare the intervention with relevant alternatives and reflect the uncertainty appropriately. In this analysis the greatest uncertainty is around the treatment effect of TAVI when compared to MM. To represent our uncertainty we have presented not one survival curve but a range of possibilities, subgroups and sensitivity analyses and in the absence of head-to-head comparisons and comparator IPD this represents the best option available. Rather than presenting a base case ICER we have used the results of the sensitivity and subgroup analyses to demonstrate just how robust the cost-effectiveness argument for TAVI vs MM is in inoperable patients.

\section{CONCLUSION}

With the low mortality and overall improvement in health-related quality of life found in the ADVANCE study, TAVI with the CoreValve system has demonstrated its value in clinical terms. With this analysis we can now consider the cost-effectiveness of the CoreValve system against other accepted therapies delivered in the UK NHS. Despite the high upfront costs of TAVI, it has proven to deliver substantial improvements in the length as well as quality of life, and while accommodating for the uncertainty in our analysis, it is likely to represent a cost-effective intervention. 
Author affiliations

${ }^{1}$ St. George's Hospital, London, UK

${ }^{2}$ ICON Health Economics, Oxford, UK

${ }^{3}$ Medtronic International Trading Sàrl, Tolochenaz, Switzerland

${ }^{4}$ University Hospital Antwerp, Edegem, Belgium

${ }^{5}$ Gemeinschaftskrankenhaus, Bonn, Germany

${ }^{6}$ University Hospital Bern, Bern, Switzerland

${ }^{7}$ University of Catania, Catania, Italy

${ }^{8}$ German Heart Center Munich, Munich, Germany

${ }^{9}$ McGill University Health Center, Royal Victoria Hospital, Montreal, Canada

${ }^{10}$ Royal Brompton Hospital, London, UK

${ }^{11}$ University Hospital Leipzig, Leipzig, Germany

Contributors SB, JE, SM, MS and AP were involved in conception, design, analysis and interpretation of the data, drafting of the manuscript and gave final approval. RB was involved in conception and design, and gave final approval. NM and NP were involved in analysis and interpretation of the data, and gave final approval. JB, UJG, PW, CT, SB and AL were involved in acquisition and analysis of the primary data, critical revision of the manuscript, and gave final approval.

Competing interests SB serves as a consultant to Medtronic and as a proctor for Medtronic and JenaValve and has received travel expenses from Edwards Lifesciences, Medtronic and Johnson \& Johnson; SM, JE, AP and MS are employees of Icon Clinical Research Ltd, an international consultancy firm and have undertaken similar analyses for a number of healthcare firms. RB is an employee of Medtronic. JB serves as a proctor for Medtronic; UJG has received consultant and lecture fees and study-related travel expenses from Medtronic and Edwards Lifesciences, and serves as a proctor for Medtronic PW has received consultant fees from Medtronic, Edwards Lifesciences and Biotronic; and remuneration for study-related travel and for developing education materials from Medtronic. NM and NP have provided consultancy services to Medtronic. AL has received consultant fees as well as study-related travel expenses and lecture fees from Medtronic, St. Jude Medical and Biosensors.

Provenance and peer review Not commissioned; externally peer reviewed.

Data sharing statement No additional data are available.

Open Access This is an Open Access article distributed in accordance with the Creative Commons Attribution Non Commercial (CC BY-NC 4.0) license, which permits others to distribute, remix, adapt, build upon this work noncommercially, and license their derivative works on different terms, provided the original work is properly cited and the use is non-commercial. See: http:// creativecommons.org/licenses/by-nc/4.0/

\section{REFERENCES}

1. Vahanian A, Alfieri O, Andreotti F, et al. Guidelines on the management of valvular heart disease (version 2012): the Joint Task Force on the Management of Valvular Heart Disease of the European Society of Cardiology (ESC) and the European Association for CardioThoracic Surgery (EACTS). Eur J Cardiothorac Surg 2012;42:S1-44.

2. Clark MA, Arnold SV, Duhay FG, et al. Five-year clinical and economic outcomes among patients with medically managed severe aortic stenosis: results from a Medicare claims analysis. Circ Cardiovasc Qual Outcomes 2012;5:697-704.

3. Leon MB, Smith CR, Mack M, et al. Transcatheter aortic-valve implantation for aortic stenosis in patients who cannot undergo surgery. N Engl J Med 2010;363:1597-607.

4. Deutsch MA, Bleiziffer S, Elhmidi Y, et al. Beyond adding years to life: health-related quality-of-life and functional outcomes in patients with severe aortic valve stenosis at high surgical risk undergoing transcatheter aortic valve replacement. Curr Cardiol Rev 2013;9:281-94.

5. National Institute of Health and Clinical Excellence. Guide to the methods of technology appraisal 2013. http://www.nice.org.uk/ media/D45/1E/GuideToMethodsTechnologyAppraisal2013.pdf (accessed 23 Jun 2013).

6. Linke A, Wenaweser P, Gerckens U, et al. Treatment of aortic stenosis with a self-expanding transcatheter valve: the International multi-centre ADVANCE study. Eur Heart J 2014;35:2672-84.
7. Kapadia S. Three-year outcomes of transcatheter valve replacement (TAVR) in "inoperable" patients with severe aortic stenosis: the PARTNER trial [abstract]. J Am Coll Cardiol 2012;60(Suppl):S17.

8. Makkar RR, Fontana GP, Jilaihawi $\mathrm{H}$, et al. Transcatheter aortic-valve replacement for inoperable severe aortic stenosis. N Engl J Med 2012;366:1696-704

9. Watt M, Mealing S, Eaton J, et al. Cost-effectiveness of transcathete aortic valve replacement in patients ineligible for conventional aortic valve replacement. Heart 2012;98:370-6.

10. Collett D. Modelling Survival Data in Medical Research. 2nd edn. Boca Raton, FL: Chapman and Hall/CRC Press, 2003.

11. Kind $\mathrm{P}$, Hardman $\mathrm{G}$, Macran S. UK population norms for EQ-5D. 1999. Centre for Health Economics (CHE), University of York. http:// www.york.ac.uk/media/che/documents/papers/discussionpapers/ CHE\%20Discussion\%20Paper\%20172.pdf (accessed 23 Jun 2013).

12. Fox M, Mealing S, Anderson R, et al. The clinical effectiveness and cost-effectiveness of cardiac resynchronisation therapy (biventricular pacing) for heart failure: systematic review and economic model. Health Technol Assess 2007;11:ix-248.

13. British National Formulary. http://www.bnf.org/bnf/index.htm (accessed 23 Jun 2013).

14. National Health Service. NHS Schedule of Reference Costs. https:// www.gov.uk/government/publications/nhs-reference-costs-2012-to2013 (accessed 23 Jun 2013).

15. Curtis L. Unit costs of health and social care. http://www.pssru.ac.uk (accessed 23 Jun 2013).

16. Orlando R, Pennant M, Rooney S, et al. Cost-effectiveness of transcatheter aortic valve implantation (TAVI) for aortic stenosis in patients who are high risk or contraindicated for surgery: a model-based economic evaluation. Health Technol Assess 2013;17:1-86.

17. Raikou M, McGuire A, Lurz $\mathrm{P}$, et al. An assessment of the cost of percutaneous pulmonary valve implantation using melody versus surgical valve replacement in patients with right ventricular outflow tract dysfunction. J Med Econ 2011;14:47-52.

18. Varadarajan P, Kapoor N, Bansal RC. Clinical profile and natural history of 453 nonsurgically managed patients with severe aortic stenosis. Ann Thorac Surg 2006;82:2111-15.

19. Bouma BJ, van Den Brink RB, van der Meulen JH, et al. To operate or not on elderly patients with aortic stenosis: the decision and its consequences. Heart 1999;82:143-8.

20. Government Actuarial Department. https://wwwgov uk/government/ (accessed 23 Jun 2013).

21. Mylotte D, Osnabrugge RL, Windecker S, et al. Transcatheter aortic valve replacement in Europe: adoption trends and factors influencing device utilization. J Am Coll Cardiol 2013;62:210-19.

22. Cribier A, Eltchaninoff $\mathrm{H}$, Bash $\mathrm{A}$, et al. Percutaneous transcatheter implantation of an aortic valve prosthesis for calcific aortic stenosis: first human case description. Circulation 2002;106:3006-8.

23. Neyt M, Van BH, Van De Sande S, et al. Transcatheter Aortic Valve Implantation (TAVI): a Health Technology Assessment Update. KCE 163C. 2011. http://kce.fgov.be/publication/report/transcatheter-aorticvalve-implantation-tavi-a-health-technology-assessment-update (accessed 24 Jun 2013).

24. Van Brabandt $\mathrm{H}$, Neyt $\mathrm{M}$, Hulstaert $\mathrm{F}$. Transcatheter aortic valve implantation (TAVI): risky and costly. BMJ 2012;345:e4710.

25. Reynolds MR, Magnuson EA, Wang K, et al. Cost-effectiveness of transcatheter aortic valve replacement compared with standard care among inoperable patients with severe aortic stenosis: results from the placement of aortic transcatheter valves (PARTNER) trial (Cohort B). Circulation 2012;125:1102-9.

26. Reynolds MR, Magnuson EA, Lei Y, et al. Health-related quality of life after transcatheter aortic valve replacement in inoperable patients with severe aortic stenosis. Circulation 2011;124:1964-72.

27. Gada H, Kapadia SR, Tuzcu EM, et al. Markov model for selection of aortic valve replacement versus transcatheter aortic valve implantation (without replacement) in high-risk patients. Am J Cardiol 2012;109:1326-33.

28. Neyt $\mathrm{M}$, Van $\mathrm{BH}$, Devriese $\mathrm{S}$, et al. A cost-utility analysis of transcatheter aortic valve implantation in Belgium: Focusing on a well-defined and identifiable population. BMJ Open 2012;2:pii: e001032.

29. Sehatzadeh S, Doble B, Xie F, et al. Transcatheter aortic valve implantation (TAVI) for treatment of aortic valve stenosis: an evidence-based analysis (part B). Ont Health Technol Assess Ser 2012;12:1-62

30. Neyt M, Van Brabandt $\mathrm{H}$. Cost-effectiveness of transcatheter aortic valve replacement: overoptimistic study results and a call for publication of complete trial results. Heart 2012;98:1031-3; author reply $1033-4$. 